\title{
CMEARTICLE
}

\section{Clinics in diagnostic imaging (185)}

Ying Xin Candice Leong ${ }^{1}$, MBBS, FRCR, Poh Lye Paul See ${ }^{2}$, MBBS, FRCR

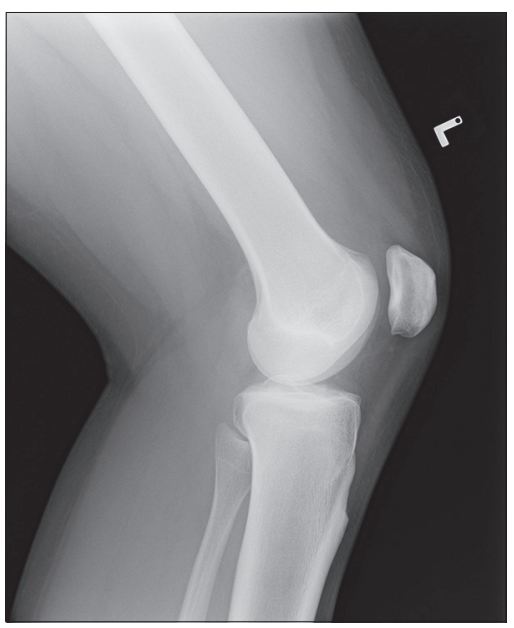

Fig. 1 Lateral radiograph of the left knee.
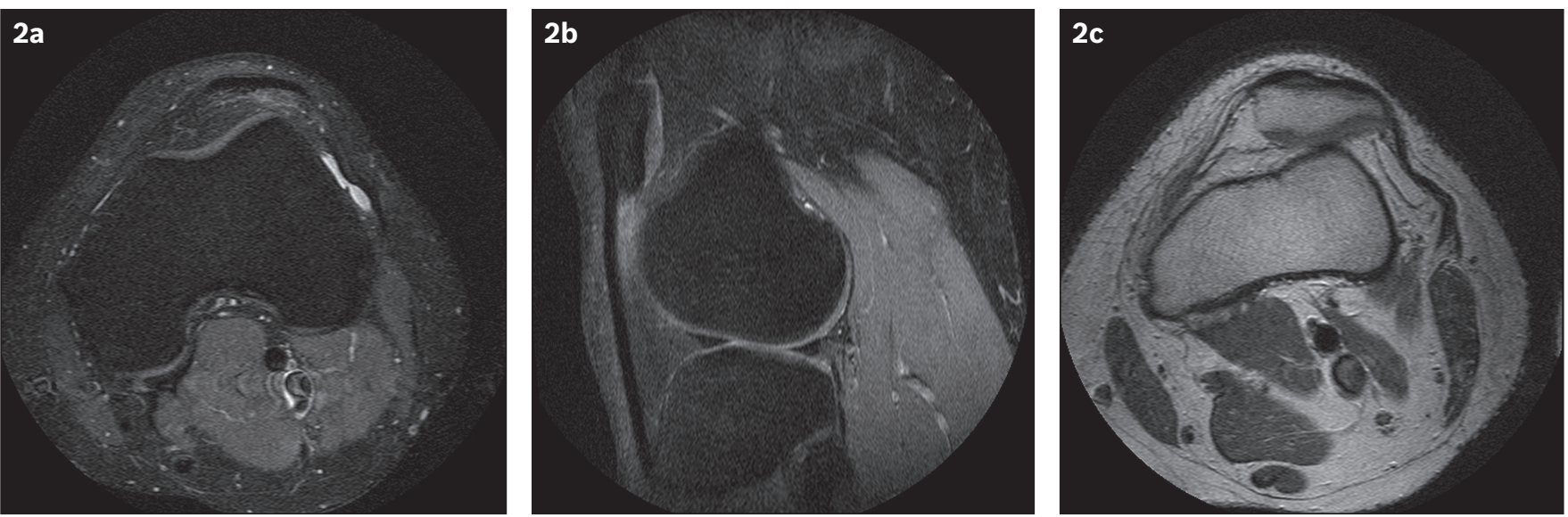

Fig. 2 (a) Axial and (b) sagittal fat-saturated proton density fast spin-echo and (c) axial proton density fast spin-echo MR images of the left knee.

\section{CASE PRESENTATION}

A 20-year-old National Serviceman presented with six weeks of left anterior knee pain and swelling after training for his individual physical fitness test. The pain was predominantly over the lateral aspect of his patella and was exacerbated by running and squatting for prolonged periods. There was also occasional clicking in the knee and the sensation of instability.
Physical examination revealed tenderness over the lateral joint line and lateral patellar edge. Although there was full range of movement of the knee, pain was elicited at extreme flexion. Patellar grind test and lateral McMurray's test were positive. What do the radiograph (Fig. 1) and subsequent magnetic resonance (MR) images (Fig. 2) of the knee show? What is the diagnosis? 


\section{IMAGE INTERPRETATION}

Lateral radiograph of the left knee (Fig. 1) shows the patella in an abnormally elevated position, resulting in a patella tendonto-patella length ratio (Insall-Salvati ratio) of about 1.6. The findings are consistent with patella alta. Axial and sagittal proton density fat-saturated fast spin-echo MR sequences (Figs. 2a \& b) of the knee show a focal area of increased signal intensity at the superolateral aspect of the infrapatellar (Hoffa's) fat pad. Patella alta was confirmed on MR imaging and lateral patellar tilt (Fig. 2c) was also noted. No other significant abnormalities were detected.

\section{DIAGNOSIS}

Patellar tendon-lateral femoral condyle friction syndrome (PTLFCFS) and patella alta.

\section{CLINICAL COURSE}

The patient was treated non-operatively with a course of physiotherapy and given advice on rest and activity modification. At his review six months later, there was reduced pain and he was able to run short distances with minimal discomfort.

\section{DISCUSSION}

Anterior knee pain is a common complaint among patients referred for MR imaging and remains a challenging problem to evaluate, diagnose and treat. This is in part due to the complex network of structures in the anterior knee compartment and the even more sophisticated system of interaction between them to achieve stable and balanced knee motion. Patellofemoral disorders are among the most frequently described sources of anterior knee pain, ${ }^{(1)}$ but the precise mechanisms and predisposing risk factors that give rise to the development of pain are not yet fully understood.

Patellofemoral pain has been classified by the Lyon school into three well-defined entities ${ }^{(2)}$ that describe a spectrum of disorders and their clinical manifestations relating to patellofemoral joint stability: (a) objective patellar instability $(\mathrm{OPI})$, which is defined by the presence of at least one episode of true patellar dislocation or radiological sequelae and at least one anatomical abnormality confirmed by imaging, such as trochlear dysplasia, patella alta, patella tilt or tibial tubercle-trochlear groove (TT-TG) abnormality; (b) potential patellar instability (PPI), characterised by the presence of subjective patellar pain and/or instability (reflex) with no history of true patellar dislocation and the same anatomical abnormalities as in OPl; and (c) patellar pain syndrome (PPS), in which no major morphological abnormality is found on radiographs. The symptoms are a combination of patellar pain, reflex buckling and, sometimes, pseudolocking.

Transient lateral patellar dislocation and its MR findings have been discussed in a previous case in this Medical Education series. ${ }^{(3)}$ We herein present a complementary case of PT-LFCFS that may fall within the definition of either PPI or PPS. PT-LFCFS is a less-recognised but important cause of patellofemoral arthralgia and an indicator of patellar maltracking and instability. ${ }^{(4)}$ As such, recognition of this entity is important, as it has implications for both diagnosis and treatment.
PT-LFCFS is a form of impingement that occurs at the superolateral aspect of the infrapatellar fat pad between the patella and lateral femoral condyle, resulting in contusion and reactive inflammatory changes within the fat. When present, this manifests on MR imaging as focal increased signal on T2weighted and fat-saturated images. The term was first used by Chung at $\mathrm{el}^{(5)}$ in a retrospective study of 42 patients with chronic anterior and lateral knee pain. Although increasingly reported, this condition remains frequently overlooked and underreported in radiological literature. ${ }^{(6)}$

The infrapatellar fat pad is an intracapsular but extrasynovial structure that is bound by the patellar tendon anteriorly and the synovial-lined tibiofemoral joint posteriorly, with further posterior extension into the intercondylar notch. The fat pad is attached to the inferior surface of the patella superiorly and extends to the level of the cartilage overlying the anterior aspect of the femoral condyles posterosuperiorly. Inferiorly, it is attached to the periosteum of the tibia and the anterior horns of the medial and lateral menisci. The infrapatellar fat pad serves to increase the surface area of the synovium and facilitates the distribution of lubricant in the joint. ${ }^{(6)}$ It receives its innervation from branches of the femoral, common peroneal and saphenous nerves, and is richly innervated by Type IVa free nerve endings, which transmit pain and inflammation, and is therefore a recognised source of anterior knee pain.

The typical presentation of PT-LFCFS is anterior knee pain inferior to the patella, which is exacerbated by knee extension. This is associated with focal point tenderness in the inferior patella. The fat pad is thickened on the affected side, resulting in difficult palpation of the patella. ${ }^{(5)}$ Both signs and symptoms are non-specific, often generating a wide range of clinical differentials, such as patellofemoral stress syndrome, chondromalacia patellae, patellar tendinopathy and tear of the lateral meniscus. ${ }^{(1)}$ This highlights the importance of MR imaging in the evaluation of patellofemoral arthralgia. On MR imaging, superolateral fat pad oedema appears to be a sensitive but non-specific sign for PT-LFCFS. In a study by De Smet et $\mathrm{al},{ }^{(7)}$ this finding was present in all clinically symptomatic patients, although it was also seen in asymptomatic patients.

The presence of anatomical predispositions contributing to instability in patients raises the index of suspicion for PT-LFCFS. They include the presence of patella alta, patellar malalignment, a TT-TG distance of $\geq 20 \mathrm{~mm}$ and trochlear dysplasia. Assessment of these conditions was discussed in the aforementioned case earlier in this series. ${ }^{(3)}$

Following the diagnosis of PT-LFCFS, non-operative management is the mainstay of treatment, with rest, activity modification and ice as the essential components. ${ }^{(8)}$ Antiinflammatory drugs often help in the initial stages of treatment to reduce pain and inflammation and improve the patient's compliance with physical therapy. Surgery is considered when conservative treatment fails to provide symptom control. The main techniques involve distal realignment, moving the tibial tubercle anteriorly and lateral retinacular release, all of which attempt to decrease the contact stresses between the patella and distal femur in the patellofemoral articulation. 
PT-LFCFS is likely related to the clinical entity that has previously been called Hoffa's fat pad impingement, described by Albert Hoffa in 1904. The latter is due to single or repetitive traumatic episodes with hyperextension and rotational forces causing haemorrhage. The inflamed fat pad then becomes hypertrophied, predisposing it to impingement between the tibia and femur, and thus, further injury and inflammation. ${ }^{(9)}$ On MR images, acute findings indicate the presence of fluid, oedema and haemorrhage (Fig. 3), and chronic findings resemble those of scarring after knee arthroscopy or surgery (Fig. 4). ${ }^{(9)}$ On T2weighted images, the acutely affected region of the fat pad may have diffuse high signal intensity, oedema or a heterogeneous appearance due to haemorrhage. The patellar tendon may be bowed anteriorly by the swollen fat pad. In chronic cases, areas of low signal intensity within the fat pad may represent foci of haemosiderin, fibrosis or ossified fibrocartilaginous tissue.

Several other entities that involve the peripatellar fat may be encountered when evaluating MR imaging of the knee. Cysts around the knee are common and those occurring within the infrapatellar fat pad may resemble Hoffa's disease. Ganglion cysts arising in the infrapatellar fat pad were previously thought to be rare, with only a few having been reported in the literature (Fig. 5). ${ }^{(10)}$ However, they are increasingly being diagnosed with the advent of MR imaging. Within this location, the pathogenesis of ganglion cysts is not fully understood, although they are thought to arise from the alar folds lined by synovium that are intrinsically present within the fat pad. The MR appearance of these ganglion cysts is similar to that of ganglia at other sites, being mainly isointense to fluid with hypointense signal on T1-weighted sequences and hyperintense signal intensity on T2-weighted sequences. They are typically multiloculated or septated, and rarely communicate with the joint. Administration of intravenous gadolinium will show enhancement of the rim of the ganglion and any septa.

A parameniscal cyst is another intra-articular cystic lesion that has a similar appearance to a ganglion cyst and may involve the infrapatellar fat pad. It can be difficult to differentiate between the two, especially when the meniscal cyst arises from the anterior horn of the meniscus and extends

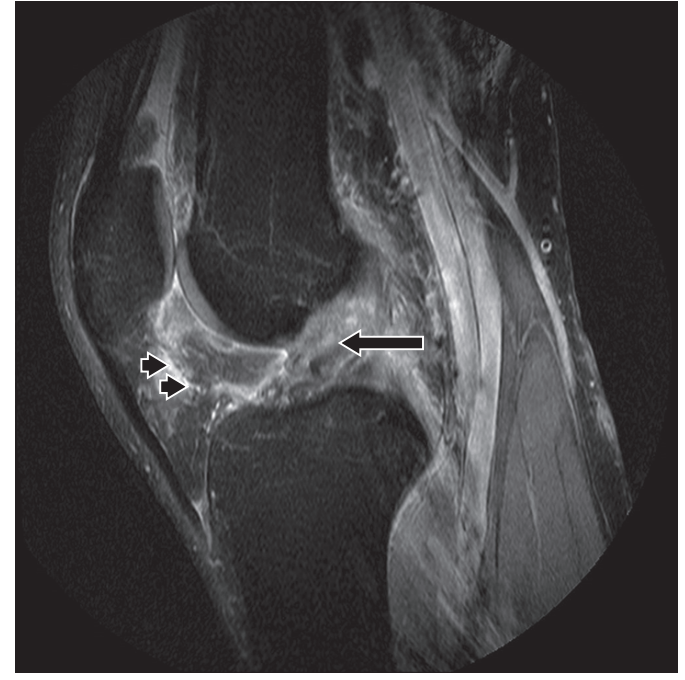

Fig. 3 A 30-year-old man sustained a twisting injury to the left knee. Sagittal fat-saturated proton density fast spin-echo MR image shows an acute shear injury of the infrapatellar fat pad (arrowheads). An accompanying complete tear of the anterior cruciate ligament (arrow) is noted.

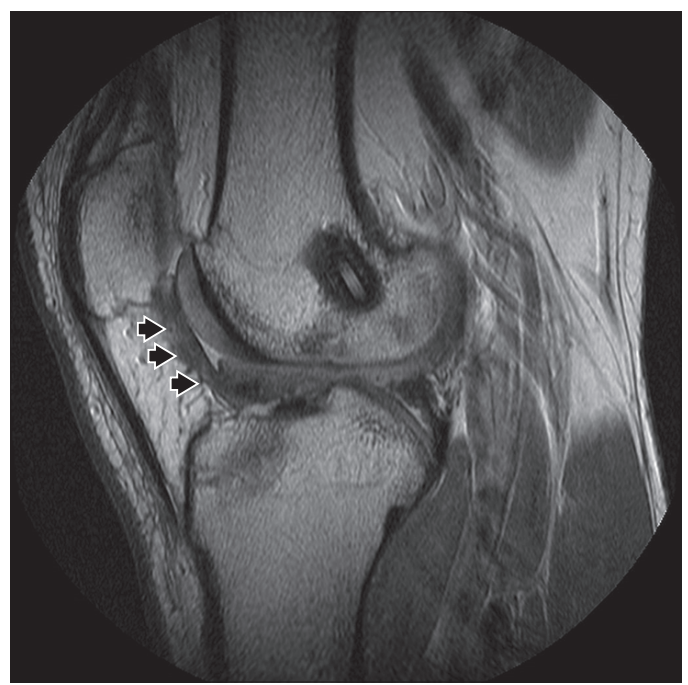

Fig. 4 A 21-year-old man who had prior anterior cruciate ligament (ACL) reconstruction presented with left knee locking. Sagittal fat-saturated proton density fast spin-echo MR image shows a prominent area of arthrofibrosis (arrowheads) anterior to the reconstructed ACL graft. Findings are related to chronic scarring due to the previous surgery.
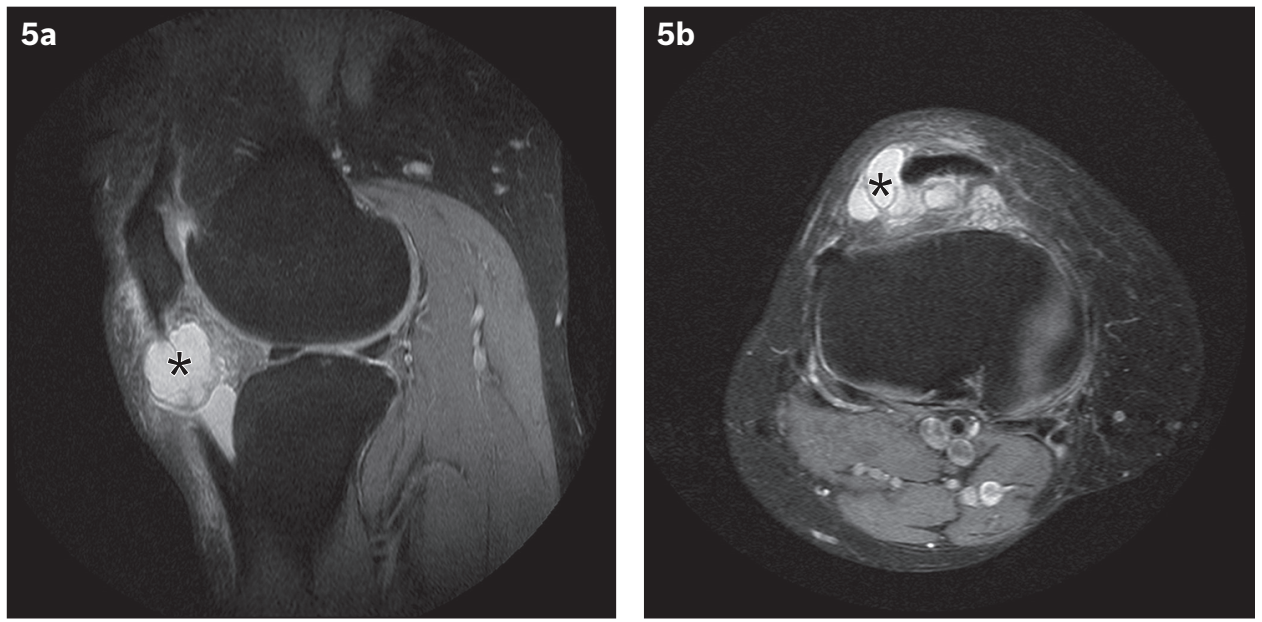

Fig. 5 A 59-year-old woman presented with localised swelling at the lateral joint line of the right knee. (a) Sagittal and (b) axial fat-saturated proton density fast spin-echo MR images show a ganglion cyst (asterisks) involving the infrapatellar fat pad. 

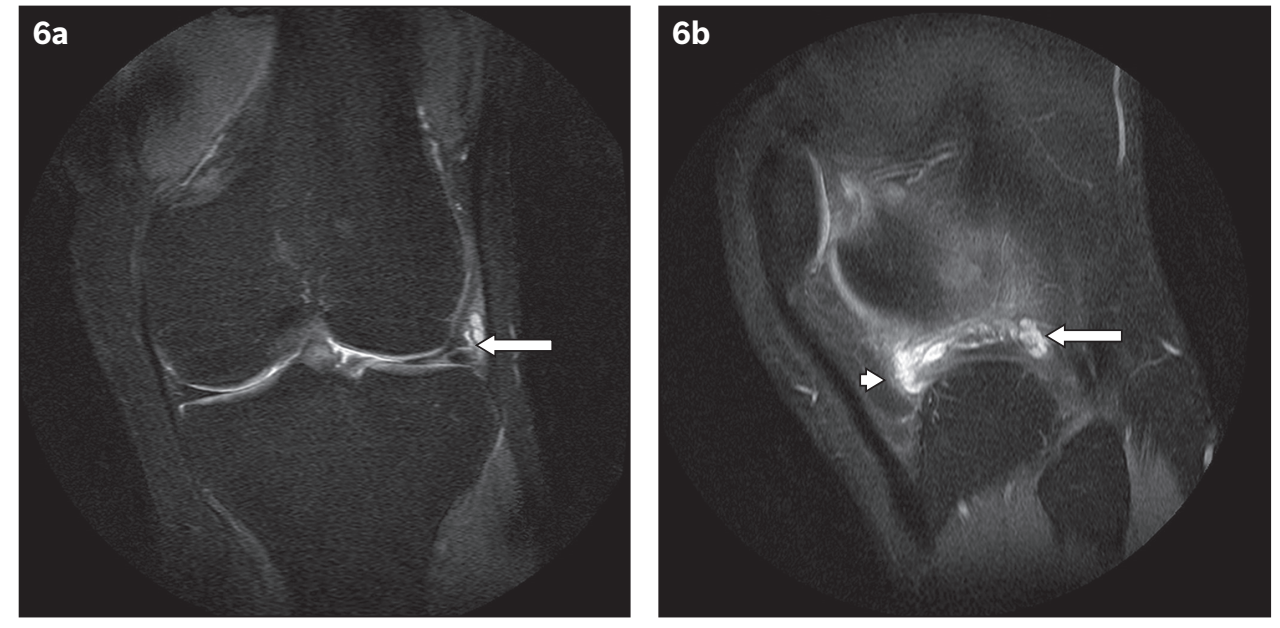

Fig. 6 A 22-year-old man presented with lateral joint line tenderness in the left knee. (a) Coronal and (b) sagittal fat-saturated proton density fast spinecho MR images show a multiloculated parameniscal cyst related to a horizontal tear of the body of the lateral meniscus (arrows), which decompresses anteriorly toward the Hoffa's fat pad (arrowhead).
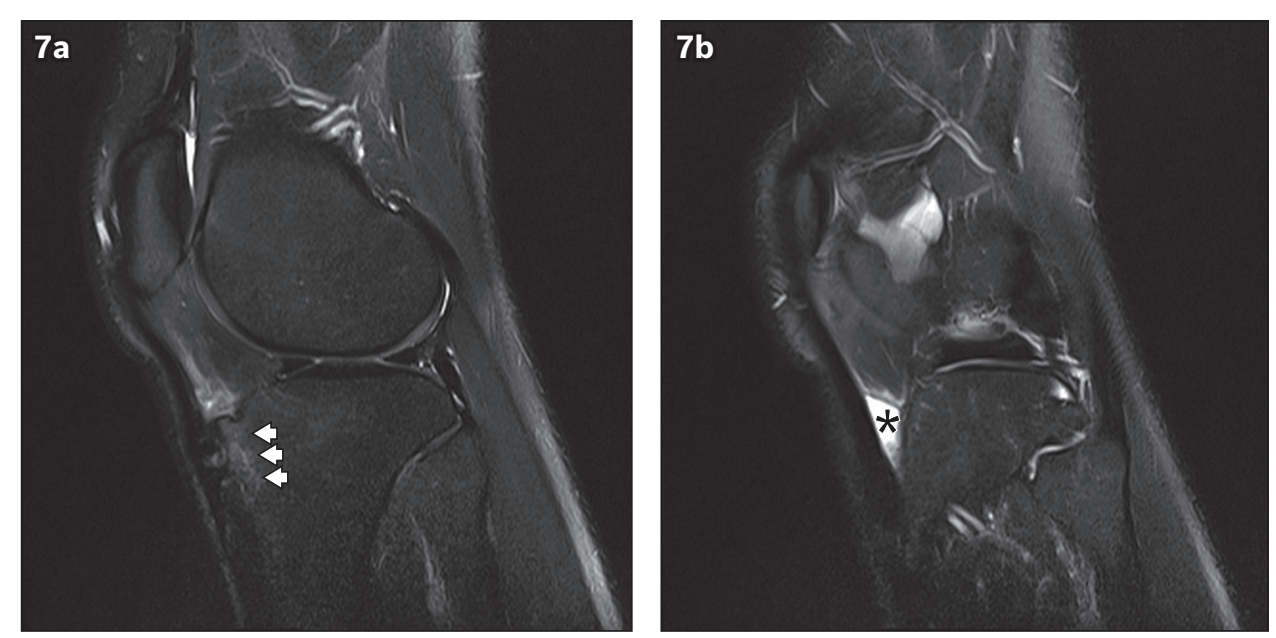

Fig. 7 A 19-year-old man presented with pain at the anterior aspect of the knee. Sagittal proton density fat-saturated turbo spin-echo MR images show (a) fragmentation and increased signal intensity of the tibial tuberosity (arrowheads) compatible with Osgood-Schlatter disease, and (b) a triangular fluid signal collection deep to the distal patellar tendon (asterisk). This is the typical appearance of deep infrapatellar bursitis.

into the infrapatellar fat pad. The clue to differentiation is the presence of a horizontal meniscal tear in the case of a parameniscal cyst (Fig. 6). ${ }^{(11)}$ Other cystic-appearing intraarticular lesions on T2-weighted sequences include synovial haemangiomas and synovial sarcomas. However, they have distinctive MR appearances and are easily differentiated from ganglion cysts, especially with the use of contrast-enhanced MR sequences.

A further, not uncommon, abnormality to occur in the infrapatellar fat pad is deep infrapatellar bursitis, also known as retropatellar bursitis. Deep infrapatellar bursae are located between the distal patellar tendon and the anterior cortex of the proximal tibia. Small fluid collections in this area can be physiologic and are therefore considered to be normal. However, when there is pain and tenderness above the level of the tubercle or a palpable mass lateral to the distal patellar tendon, the diagnosis of infrapatellar bursitis should be considered. Deep infrapatellar bursitis usually results from overuse of the knee extensor mechanism, especially in runners and jumpers. ${ }^{(12)}$ It has also been identified in patients with Osgood-Schlatter disease. In one study of such patients, a distended deep infrapatellar bursa was seen on MR imaging in $71 \%(12 / 17)$ of cases. ${ }^{(13)}$ Deep infrapatellar bursitis is also associated with infection and inflammatory conditions. On MR imaging, it appears as a triangular fluid collection posterior to the patellar tendon (Fig. 7).

In conclusion, PT-LFCFS is a less well-recognised but important cause of anterior knee pain and represents an entity in a spectrum of disorders related to patellofemoral instability. It is also one of several conditions that affect the infrapatellar fat pad, with specific findings on MR imaging. Although the conditions are related based on the location of the disease, their imaging appearances are distinctly different, allowing differentiation of the abnormalities, which thereby enables the clinician to provide the necessary and appropriate care based on the correct diagnosis. 
ABSTRACT A 20-year-old National Serviceman presented with left knee pain and swelling after training for his physical fitness test. Lateral knee radiography and magnetic resonance (MR) imaging showed patellar tendon-lateral femoral condyle friction syndrome (PTLFCFS), on a background of patella alta and patellar malalignment. The patient was treated non-operatively with a course of physiotherapy and given advice on rest and activity modification. PT-LFCFS is a less wellrecognised but important cause of anterior knee pain and represents an entity in a spectrum of disorders related to patellofemoral instability. We herein discuss the MR imaging findings specific to and associated with this condition, as well as briefly describing treatment options. In addition, we showcase a range of commonly encountered abnormalities that affect the infrapatellar fat pad and briefly discuss their specific MR imaging findings.

Keywords: anterior knee pain, maltracking, patella alta, patellar tendon-lateral femoral condyle friction syndrome, trochlear dysplasia

\section{REFERENCES}

1. Biedert RM, Sanchis-Alfonso V. Sources of anterior knee pain. Clin Sports Med 2002; 21:335-47.

2. Dejour H. [Instabilities of the patella]. Encycl Med Chir, Locomotor Device. Paris: Elsevier, 1996; 14-328-A-10:8p. French.

3. Zhang J, Lee $\mathrm{CH}$. Clinics in diagnostic imaging (163). Transient lateral patellar dislocation with trochlear dysplasia. Singapore Med J 2015; 56:542-8.

4. Subhawong TK, Eng J, Carrino JA, Chhabra A. Superolateral Hoffa's fat pad edema: association with patellofemoral maltracking and impingement. AJR Am J Roentgenol 2010; 195:1367-73.

5. Chung CB, Skaf A, Roger B, et al. Patellar tendon-lateral femoral condyle friction syndrome: MR imaging in 42 patients. Skeletal Radiol 2001; 30:694-7.

6. Saddik D, McNally EG, Richardson M. MRI of Hoffa's fat pad. Skeletal Radiol 2004; 33:433-44

7. De Smet AA, Davis KW, Dahab KS, et al. Is there an association between superolateral Hoffa fat pad edema on MRI and clinical evidence of fat pad impingement? AJR Am J Roentgenol 2012; 199:1099-104.

8. McCarthy MM, Strickland SM. Patellofemoral pain: an update on diagnostic and treatment options. Curr Rev Musculoskelet Med 2013; 6:188-94.

9. Jacobson JA, Lenchik L, Ruhoy MK, Schweitzer ME, Resnick D. MR imaging of the infrapatellar fat pad of Hoffa. Radiographics 1997; 17:675-91.

10. Amin M, Torreggiani W, Sparkes J. Infrapatellar ganglion that developed from infrapatellar fat and had minimal intraarticular extension. Knee Surg Sports Traumatol Arthrosc 2008; 16:179-81.

11. Kim MG, Kim BH, Choi JA, et al. Intra-articular ganglion cysts of the knee: clinical and MR features. Eur Radiol 2001; 11:834-40.

12. Marra MD, Crema MD, Chung M, et al. MRI features of cystic lesions around the knee. Knee 2008; 15:423-38.

13. Rosenberg ZS, Kawelblum M, Cheung YY, et al. Osgood Schlatter lesion: fracture or tendonitis? Scintographic, CT and MR imaging features. Radiology 1992; 185:853-8. 


\section{SINGAPORE MEDICAL COUNCIL CATEGORY 3B CME PROGRAMME} (Code SMJ 201804A)

Question 1. Regarding patellar tendon-lateral femoral condyle friction syndrome (PT-LFCFS):

a) It is one of the causes of anterior knee pain.

b) It is an indicator of patellar maltracking and instability.

c) It can cause patellar dislocation.

d) It is due to impingement of the inferomedial aspect of the infrapatellar fat pad between the patella and the lateral femoral condyle.

Question 2. The following are imaging features that may be present in PT-LFCFS:

a) Anterior bowing of the patellar tendon.

b) Areas of T2-weighted low signal intensity within the infrapatellar fat pad.

c) A tibial tubercle-trochlear groove distance of equal to or greater than $20 \mathrm{~mm}$.

d) Oedema of the patellar tendon.

Question 3. Concerning the diagnosis of PT-LFCFS:

a) Superolateral fat pad oedema is a specific finding on magnetic resonance imaging.

b) Imaging is often unnecessary to make the diagnosis.

c) Surgery is recommended as the first line of treatment.

d) It is associated with patella alta.

Question 4. The following are differentials for cystic lesions of the infrapatellar fat pad:
a) Synovial sarcoma.
b) Haemangioma.
c) Ganglion cyst.
d) Infrapatellar bursitis.

Question 5. Concerning the infrapatellar fat pad:

a) It is an extracapsular and intrasynovial structure.

b) It receives innervation from branches of the femoral, common peroneal and saphenous nerves.

c) It is attached to the anterior horns of the medial and lateral menisci.

d) It serves to increase the surface area of the synovium and facilitates the distribution of lubricant in the joint.

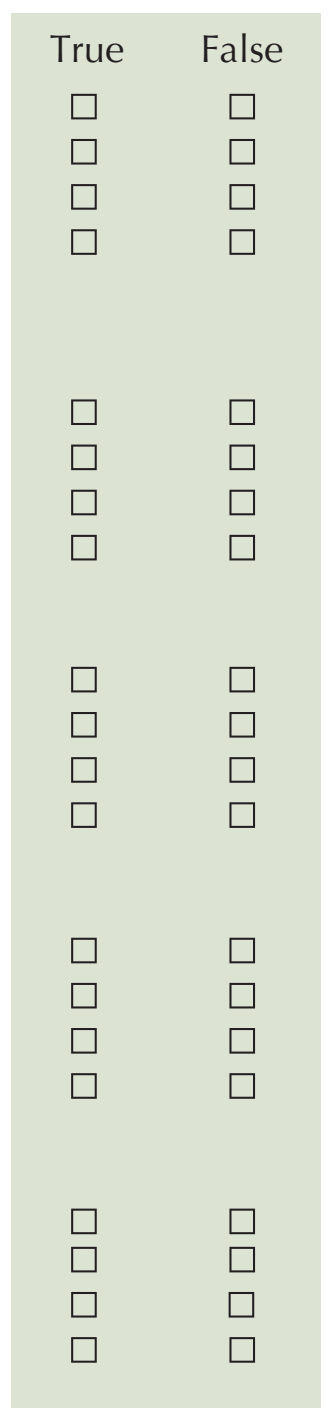

\section{Doctor's particulars:}

Name in full:

MCR no:

Specialty:

Email:

\section{SUBMISSION INSTRUCTIONS:}

Visit the SMJ website: http://www.smj.org.sg/current-issue and select the appropriate quiz. You will be redirected to the SMA login page.

For SMA member: (1) Log in with your username and password (if you do not know your password, please click on 'Forgot your password?'). (2) Select your answers for each quiz and click 'Submit'.

For non-SMA member: (1) Create an SMJ CME account, or log in with your SMJ CME username and password (for returning users). (2) Make payment of SGD 21.40 (inclusive of 7\% GST) via PayPal to access this month's quizzes. (3) Select your answers for each quiz and click 'Submit'.

RESULTS:

(1) Answers will be published online in the SMJ June 2018 issue. (2) The MCR numbers of successful candidates will be posted online at the SMJ website by 7 June 2018. (3) Passing mark is $60 \%$. No mark will be deducted for incorrect answers. (4) The SMJ editorial office will submit the list of successful candidates to the Singapore Medical Council. (5) One CME point is awarded for successful candidates. (6) SMC credits CME points according to the month of publication of the CME article (i.e. points awarded for a quiz published in the December 2017 issue will be credited for the month of December 2017, even if the deadline is in January 2018).

Deadline for submission: (April 2018 SMJ 3B CME programme): 12 noon, 31 May 2018. 